\title{
Managing Accessibility - The Configurational Approach to the Inclusive Design of Urban Spaces
}

\author{
Valerio Cutini \\ Department of Civil Engineering, University of Pisa, Pisa, Italy
}

\begin{abstract}
This paper concerns the theme of the universal accessibility to urban spaces, proposing the contribution of configurational approach to their inclusive design. Born in the first Nineties on the roots of the efforts and the studies for reducing material and architectural barriers for disabled people, such discipline extends its sphere and purposes so as to mitigate the effects of spatial exclusion of a wider and wider variety of human categories, up to virtually include any potential urban user. Several questions persuade that a merely functional approach, essentially based on the position of the located activities, cannot be said satisfying: only a configurational approach can account for the effects of the grid configuration on the actual accessibility of its spaces. Our research focuses on Italian urban settlements, which appear as ideal case studies, due to the typical consistency of their urban spaces, which, especially in the inner historical cores, are densely build, geometrically irregular and hence generally far from actually providing a universal material accessibility. Here the impedance of space is much more than a theoretical hypothesis and concrete interventions are generally requested to eliminate or reduce its barriers and to make space actually and safely usable. Rather than at indicating the technical solution of single urban problems, which of course are strongly different each other, the research aims at defining a method suitable for any local context. Such method, integrating into a reliable tool the configurational vision with the functional and interactional approach, will provide a hierarchy of urban spaces with reference to the necessity of their universal accessibility.
\end{abstract}

Keywords: Accessibility, configurational analysis, inclusive design, town planning.

\section{Introduction}

The universal accessibility of the urban spaces is one of the themes that in the recent years has gone attracting a growing attention: the progressive ageing of the population as well as the increasing expectation of a normal fruition of buildings, spaces and functions from people of all conditions involve the pressing need of modifying spaces and paths, in order to upgrade their actual level of material accessibility. The growth of an exigency like that goes matching with the cultural development around the approach to the theme of disability and accessibility. Once, around the first 80 s, such matter did actually coincide with the theme of the removal of the architectural barriers, and essentially consisted in the efforts for reducing the material obstacles that physically keep the disabled people from

Corresponding author: Valerio Cutini, $\mathrm{PhD}$, professor, research fields: town planning, urban modeling, spatial analysis, configurational analysis. E-mail: valerio.cutini@ing.unipi.it. the actual use of the space, so as to discriminate or even to exclude them from social life and human relations. It really was a philanthropic kind of approach, and, as a matter of fact, it has gone providing remarkable results, driving the governments of several countries all over the world to issue laws and regulations aimed at imposing a minimum threshold, consisting in an acceptable level of accessibility of some categories of buildings (such as public offices, public services, restaurants and hotels). Among them, we can mention the Americans with Disabilities Act (ADA) of 1990 in the United States [1], recently modified by the Americans with Disabilities Act Amendments Act (ADAAA) of 2008, the Disability Discrimination Act of 1992 in Australia and the Disability Discrimination Act of 1995 in the United Kingdom. As time has gone by, the theme has gone growing up and modifying this kind of approach, also thanks to the overcoming of a strict interpretation of the definition of disability. As a 
consequence, the discipline has extended its sphere and purposes from the mere protection of disabled people towards the mitigation of the effects of spatial exclusion of a wider and wider variety of human categories: we obviously still refer to disabled people, but also to elderly people, weak and sick persons (such as, for instance, heart patients and obese persons), and even to transitory invalids and occasionally impaired persons (such as injured persons and pregnant women) as well as children, up to virtually include any potential urban user; what hence provides the term accessibility with the attribute universal. The language in fact has gone underlining this aspect, substituting in the common speaking the previous terms barrier-free or disabled accessibility with other notions (substantially equivalent each other), such as inclusive design, design for all, universal design, integral accessibility. Summing all up, making a space universally accessible means to make it usable without any limitation by every kind of people, so that each person, regardless of his age, sex, social class and specific condition, can be free of any obstacle that may exist in buildings, transportation terminals, sidewalks, paths, roads and vehicles, and then normally behave and feel free from any spatial restriction and discrimination in his daily life and relations [2]. In the last decade several operators, foundations and institutions have been working on this issue, promoting cultural development, scientific researches, information, and concrete interventions on the theme.

Indeed this new kind of approach has given a stimulus to a large amount of studies on the theme, which have gone generating a wide set of architectural solutions, suitable to solve (or, at least, to acceptably mitigate) a large quantity of problems regarding the accessibility of urban and architectural space. As an example, for what specifically concerns the open urban spaces, solutions have been worked out and largely experimented for eliminating the steps in the sidewalks and in the accesses to the buildings, for easily accessing to parking and recreational areas, for safety crossing vehicular roads, for getting over differences in height, drops and slopes, for choosing the correct size and paving materials of sidewalks and pedestrian paths, and so on. A detailed treatment of such solutions, that appear irrelevant to the purposes of the present research, can easily be found in the wide specific literature (in particular see Refs. [2-7]).

If then the question regarding the technical way of enhancing the spatial accessibility can be said plainly set up, at the moment what concerns the actual realization of the interventions is instead far from an acceptable solution. The efforts so far appear in fact essentially concentrated within the most prominent public activities, and are aimed at upgrading their inner accessibility; in such sense, laws and regulations in several countries are getting more and more strict for what concerns the inner architectural space, extending instructions and duties even to private residential buildings. On the contrary, the grid of the paths which connect them and all the open spaces mostly remain an unexplored field, and its elements are still far from being regarded as universally accessible. The question, of course, is mainly an economic matter: the intervention for modifying the public open space in order to make it accessible would be extremely expensive, due to the large extension of the whole grid, to the large amount of any kind of obstacles and to the frequent presence of constraints and limitations. In Italy, these problems appear particularly difficult to face, due to the irregular orographic consistency of a large part of the urban settlements, which, often located on the steep hills, can hardly be made actually accessible. Beside, most of the Italian settlements have grown around an inner ancient core, often still enclosed within the original town walls, which is densely and irregularly build and characterized by tortuous and narrow paths: here, where most of the prominent urban activities are located, the intervention for the inclusive design of public open spaces would be very expensive and subjected to several architectural duties and limitations. Working on the theme of the physical 
accessibility of urban space, Italian settlements hence appear as ideal case studies, because here problems and difficulties often result so magnified as to be assumed as paradigmatic. Here the conditions of the context hinder the pedestrian movement by several kinds of material barriers and make the spatial impedance much more than a theoretical assumption or a conceptual notion; here the universal accessibility is a hard challenge to face and then an expensive target to gain.

These obvious remarks therefore lead to assume the universal accessibility of the whole urban grid as an asymptote, and hence to regard each effort for reducing exclusion and segregation as a step towards such ideal goal. Basing on the consequent evidence that the whole space of a settlement cannot be made all equally and universally accessible, a fundamental question does hence arise: which paths should be reclaimed from any possible material obstacle? In other words, the matter regards the way of selecting, within the whole grid of an urban settlement, the parts that ought to be interested by an intervention aimed at making them universally accessible. More in general, the question is the way of calibrating the actual level of physical accessibility requested by each single part of a settlement, from the lowest degree up to its highest grade, that is the universal accessibility; and this question is anything but obvious, as well as essential for avoiding to waste resources and for the optimal distribution of the available budget. In Italy, the first laws regarding the removal of architectural barriers date back to 1989, when two acts (L. nr. 13/1989 and DM nr. 236/1989) essentially imposed a minimum standard of accessibility to the inner space of new buildings. Later, a specific kind of urban plan, named PEBA (Piano per l'Eliminazione delle Barriere Architettoniche, or Plan for Architectural Barriers Elimination) was established by other acts (L. nr. 104/1992 and DPR nr. 503/1996), aimed at extending to the urban spaces an acceptable level of accessibility. Such plan was specified in several Italian regions by the local laws, and in Tuscany, one of the keenest regions on the theme of disability, a specific act was passed in 1991, imposing the PEBA to each local municipality. Nonetheless, at present the guidelines for the construction of those plans have not yet been passed, just few PEBAs have been worked out, and the presented ones appear far from being satisfying, in that they are limited at monitoring and managing the actual degree of the present material accessibility of the most used paths; when, rarely, some concrete intervention is provided, this is selected on the mere basis of a discretionary and intuitive evaluation. It's then clear that an objective method for calibrating the need of accessibility in the urban grid is definitely advised and highly requested, in order to make actual the respect of the existing laws and to avoid the waste of the available resources. Although obvious, it's worth pointing out that an effort like this would be unnecessary if only the local conditions and the economic resources could allow to plainly extend a universal accessibility all over the grid; what unfortunately everywhere, but in particular in Italy, is still so far from happening.

\section{The Methodological Approach}

The easiest and most frequent approach to the question is functional: since the most prominent public activities ought to be located in accessible buildings, also the paths that connect them (at least the shortest ones or, even better, the most used and then crowded) should hence be made universally accessible. Several faults are hidden behind this apparent solution. First, which activities along the paths of the grid such accessible paths should connect? Beside, activities can be easily and frequently moved or displaced: what then about the effects of their possible shifting on the accessibility of the connecting paths? And, if we assume to preferably connect the most relevant punctual activities, what about the diffused ones (for instance shops, offices and minute services), that are atomized and capillarity scattered along the paths of the urban grid? And, finally, what about the residential function, which in most cases appears homogeneously 
distributed all over the settlement and does generate a large amount of the origin destination movements to all the other activities? All these questions are hard to answer, and make hence evident that a merely functional approach, essentially based on the presence and the position of the located activities, cannot be said satisfying, since it cannot reliably identify the most used and crowded paths in order to assure them a universal accessibility. In other words, and more in general, the grid of the urban paths cannot be seen as the mere inactive stage where activities mutually interact; space does matter, in that its configuration affects the way the space itself is actually used. This remark induces to set aside the located activities and to focus on the urban grid, to be seen as a "machine for maximizing contacts" and interactions [8]; the analysis of its configuration will then be expected to provide significant results with reference to the likely use of the urban space. Such results could possibly be integrated with the results of a traditional interactional analysis, based on the functional consistency of the settlement: the urban grid with its configuration in a one hand and, in the other hand, the activities with their position and their consistency will then allow a calibration of the relevance of the paths with reference to their actual use and hence the opportunity of assuring them an acceptable level of material accessibility.

In the last decade, a remarkable amount of researches have been applied to test the actual reliability of the configurational approach. Studies [9] did verify the existence of a narrow correspondence between the configurational indices (in particular the integration value) with the pedestrian movement rates; further studies [10] did then verify also a strong correlation of the same configurational parameters with the actual presence of the located activities. As a matter of fact, these researches and others have proved the techniques of configurational analysis as a reliable tool for reproducing the distribution of the attractiveness towards the activities, and hence the distribution in a whole urban settlement of the level of centrality, if we define it in terms of appeal for the location of activities. Summing all up, on such basis we may assert the integration value as an indicator of the pure accessibility, meaning by the attribute pure the independence of its value from the presence and the position of the located activities. In other words, the integration value represents in the configurational vision what the economic-spatial potential is in the interactional approach. The economic-spatial potential of a location $a$ is analytically described by expressions like the following ones:

or

$$
\mathrm{Ea}=\mathrm{K} \Sigma \mathrm{j} \omega \mathrm{j} \text { daj }{ }^{-\gamma}
$$

$$
\mathrm{Ea}=\Sigma \mathrm{j}^{\alpha} \exp (-\beta \mathrm{caj})
$$

respectively according to a traditional gravitational approach [11, 12] or to an entropic vision [13], where $\omega_{j}$ is the size of the $j$ activity, $d_{a j}$ its distance from the location and $c_{a j}$ the respective movement cost. The economic-spatial potential is also commonly called generalised accessibility, since this kind of accessibility is here computed on the basis of the activities which are actually present, without the external and axiomatic assumption of a centre, traditionally defined [14] as the most desirable location for any kind of activity. Therefore it appears clear that the generalised accessibility (i.e., the economic-spatial potential) and the pure accessibility (i.e., the integration value) respectively represent in the interactional approach and in the configurational one the specific indicator of the relevance of each part of a settlement as an attractor (of movements and activities). It is singular (and significant) the apparent recurrence, with different meanings, of the notion of accessibility: defined in classic modeling as the opposite of the spatial impedance in the relations between activities, and then depending on their distribution (generalized accessibility), assumed in the configurational vision as determined by the configuration of the urban grid (pure accessibility) [15], appraised in the inclusive design as the possibility of using the space without any limitations (universal accessibility). Linking all up, the 
idea at the base of this research may be summarized saying that the distribution of both pure accessibility and generalised accessibility, integrated and taken together, can usefully indicate for each part of a settlement the specific opportunity of providing it with a material universal accessibility.

\section{Our Case Study}

Pisa has been selected as our specific case study, in order to apply a configurational approach to support and guide the construction of a PEBA. Several particular features concur in making Pisa an excellent urban laboratory for this kind of test. First, the size of the settlement: Pisa has got a population of around 100,000 inhabitants, what make it big enough for the significance of the results, and small enough for exhausting the analysis and complete the processing of the method. Second, the presence of a large (when compared to the extension of the whole settlement) historic core, whose origins date back to the XIII century and is at present still substantially unchanged; though a large part of the most prominent activities is still within this core, yet its geometric and morphological features make of course buildings and open spaces full up with obstacles and then hardly accessible. Besides, the presence of an enormous (again, comparatively) University: side by side with the local inhabitants, more than 50,000 students and about 2,000 teachers and researchers live in Pisa, giving life to an articulated network of interactions and movements to the University sites, which of course ought to be made easily accessible to anybody; a large amount of strong attractors does hence characterize the settlement. Finally, the presence of some highly relevant tourist points of interest, and among them mainly the leaning tower and its monumental adjacencies: millions visitors are annually attracted here from all over the world, and of course also this mighty attractor ought to be made universally accessible.

With this purpose, we have focused on the present urban grid of Pisa, as it can be drawn out of the CTR
(Carta Tecnica Regionale, the 1:2,000 planimetric base representation of the regional territory provided by the Tuscan regional administration) setting aside any other material object except the mere perimeter of the public spaces, that is those which can be used without any limit by everyone. The urban grid was then processed by Depthmap software, submitting its axial map, consisting of around 1200 lines, to axial analysis. Such processing did provide as a result a complete representation of the distribution of the configurational indices in the lines of the axial map, and, what's most relevant for our purposes, the distribution of the integration value all over the grid. As we have seen above, in fact, integration reproduces the distribution of the levels of attractiveness towards activities, and hence the distribution of centrality within an urban settlement. The distribution of global integration in the lines of Pisa, as it results from Depthmap processing, is here shown in Fig. 1.

Furthermore, the same analysis provided the distribution of local integration (that is radius 3 integration) in the lines of the grid of Pisa. Of course, those two analyses actually provided far different results: the representation of global integration, in fact, shows an evident centripetal distribution, with the strongest integrators materialized by the prevalent north-south axis, correspondent to the streets Borgo Stretto and Corso Italia, and in several orthogonal streets. These, as well as several less important streets which surround them, materialize the present integration core of the whole settlement, which is here represented in Fig. 5. A completely different layout arises from the representation of the distribution of radius 3 integration, presented in Fig. 2: here several strong local integrator appear, variously scattered in the urban grid from its inner core up to the far edge of the settlement; on the contrary, the lines resulting main global integrators in Fig. 1 still maintain high values of radius 3 integration in Fig. 2 and hence their strength as main local integrators.

With reference to the theme of centrality and hence 


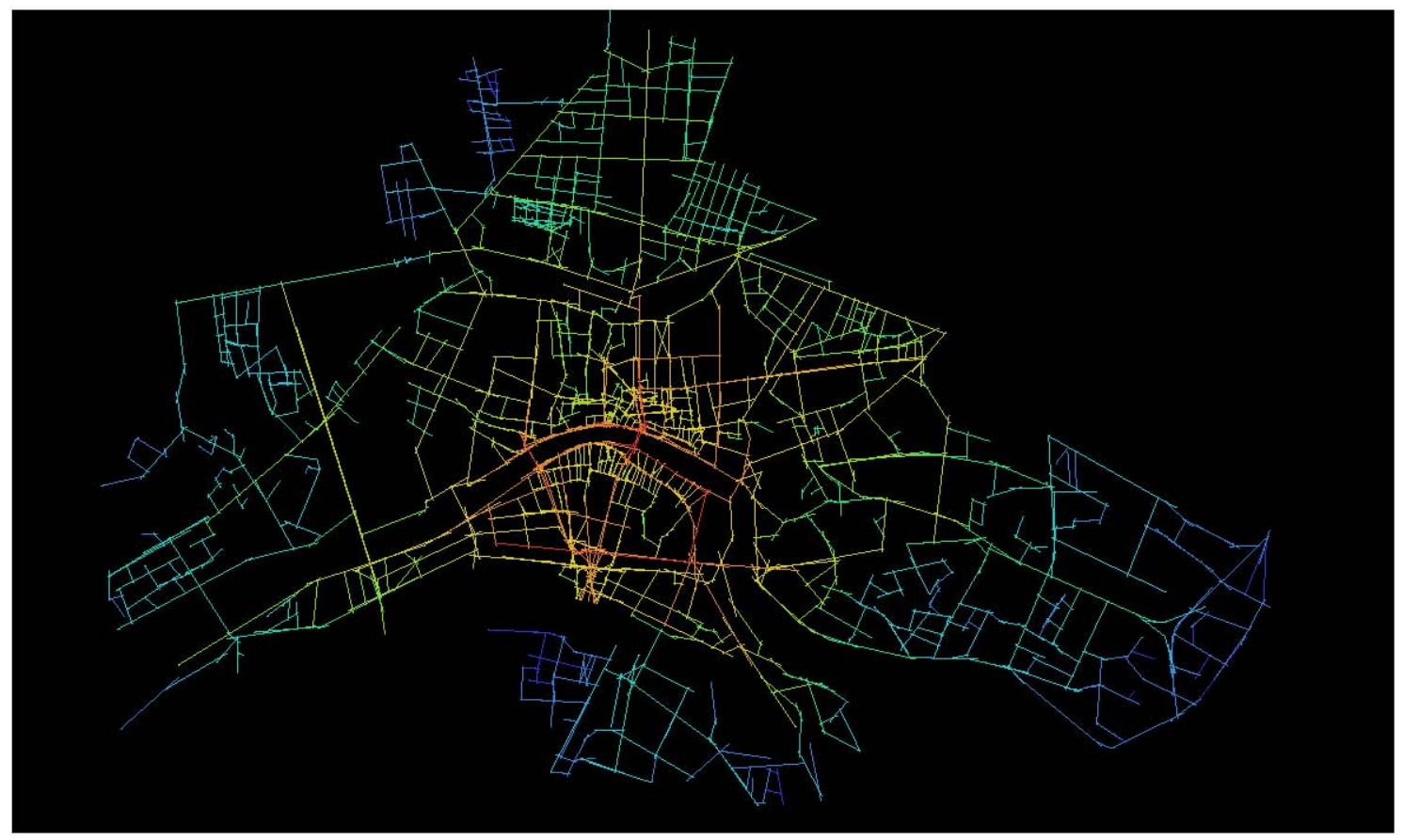

Fig. 1 Chromatic representation of the distribution of global $(R=n)$ integration in the urban grid of Pisa.

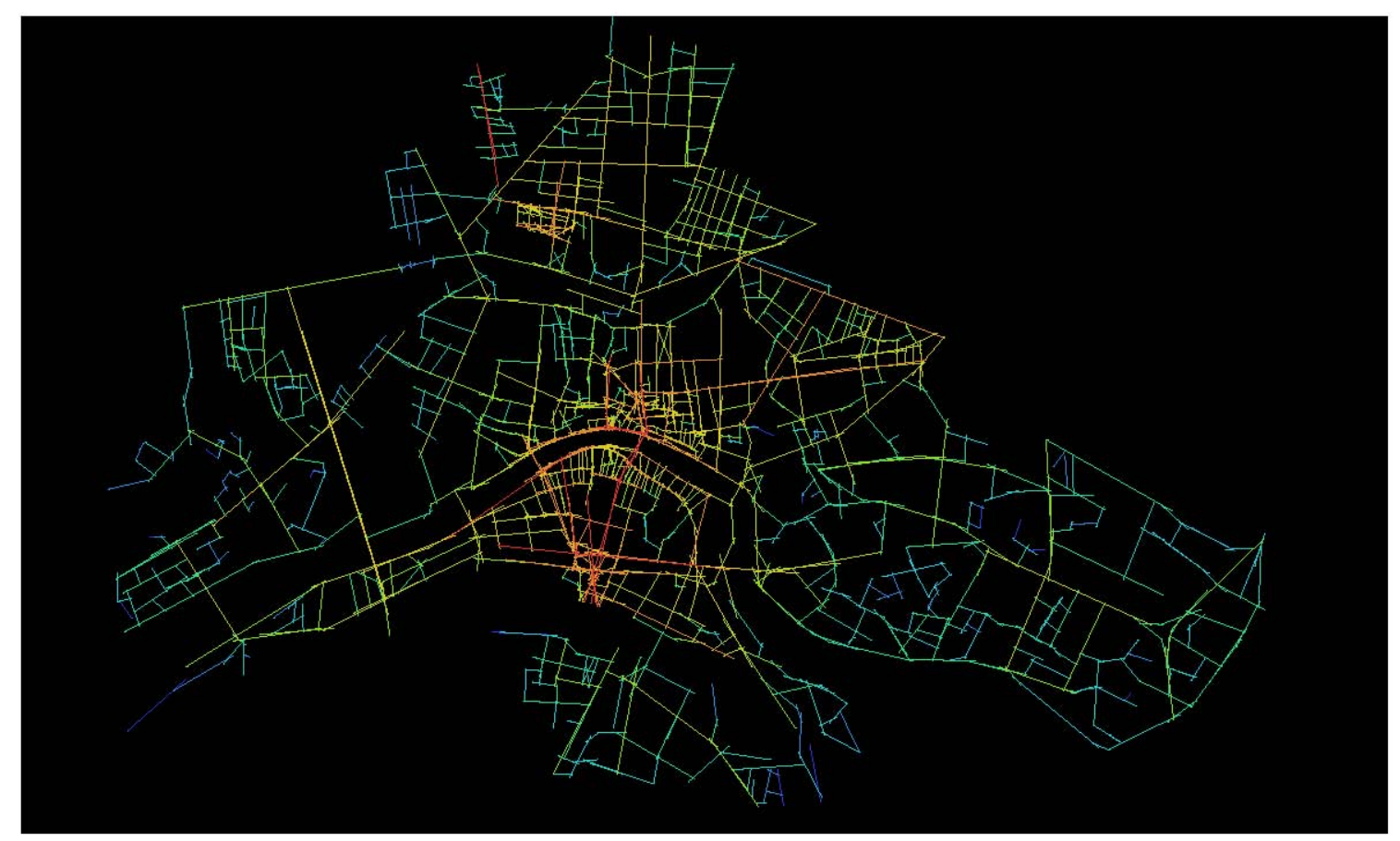

Fig. 2 Chromatic representation of the distribution of local $(R=3)$ integration in the urban grid of Pisa.

to the functional pattern of the settlement, the researches so far allow to interpret such a radical discordance and the different significance of global and local configurational values: the global integration, as we have exposed above, was proved [16] able to describe the level of centrality of each part of a small size settlement; all the same, if we analyze the whole urban area of a bigger settlement, we may notice that correlation of activities versus global integrations does weaken, while, within limited infra-urban areas, we 
observe a narrow correlation of the same activities versus the local integration value. In Pisa, we have selected 120 lines and we have observed the activities actually located in the correspondent streets; the results of the analysis of the correspondence of radius 3 integration and density of activities shows that a strong correlation connect them within local sub-systems; assuming each of them as defined by the lines gravitating towards a strong local integrator, in Pisa we have identified 10 sub-system, and then we have analyzed 10 different (but analogue) correlations. In other words, the observed correlations seems to reproduce the presence of several sub-systems of the grid; the correlation of activities versus local integration, that weakens if extended beyond each sub-system, is on its turn strongly influenced by the distribution of global integration. Summing all up, we may observe that in each sub-system the local integration value IR3 and the density of activities A appear narrowly correlated according to the following expression

$$
\mathrm{A}=\mathrm{K} \exp \mathrm{WIR}_{3}
$$

where the coefficients $\mathrm{K}$ and $\mathrm{W}$ stands for the effects of the global centrality (due to several elements: among them, the global integration value, the presence of strong global attractors, the demographic density of the area and the presence of relevant utilities and amenities) of the integrator of the sub-system on that correlation. As a consequence, the expression above can be represented by mean of several exponential curves, and each of those curves is referred to the correspondent sub-system, around its own local integrator. Other conditions being equal, the higher is the global integration value of that integrator, the higher in the (IR3, A) graph we will find the correspondent curve. Roughly speaking, a clear layout can most likely account for this correspondence: an urban settlements characterized by several local centres, with different levels of global relevance, and hence of global attractiveness; and within the area surrounding each of them, a narrow correlation between radius 3 integration and attractiveness, as materialized by the present density of activities. In such view, different kinds of activities are likely to populate the different level centres: common activities (that is activities which are not worth long distance movements, such as retail shops) mainly directed to the "peripheral" centres, rare activities (that is addressed to a wider market area and characterized by a wider range) mainly centre oriented, that's to say strongly attracted by to the "central" centre. In Fig. 3, we may observe the representation of the exponential curves local integration versus activities in the defined subsystems, where each of them is identified by a specific colour.

It can also be argued that pedestrian movements, as mainly short and exhausting within small distances, take place in a local urban context and then are more narrowly described in their distribution by the radius 3 integration rather than by the global one. Down to the matter of this research, and if we assume the location of activities as influenced by the distribution of natural movement and producing an attracted one, it's evident that the efforts for reducing any material obstacle and for providing the public open spaces with a universal accessibility ought to be oriented towards the most attracting urban areas, as the most likely to generate and receive to and from movement from all over the grid. And such purpose should be pursued assuring accessibility both to the global integration core of the urban settlement and to the local ones.

The obvious limit of this approach is the presence of non-configurational activities, which are activities that do not necessarily follow the configurational pattern of the grid. Among them, by far the most frequent and relevant are the monopolistic activities, in that those activities can be located setting aside the distribution of the configurational parameters, since they won't suffer any damage in case of a non-appealing location [17]. Many kinds of activities share this enviable feature: among them, all the public services (schools, universities, hospitals, administrative offices), but also the public amenities (parks, museums, monuments and 


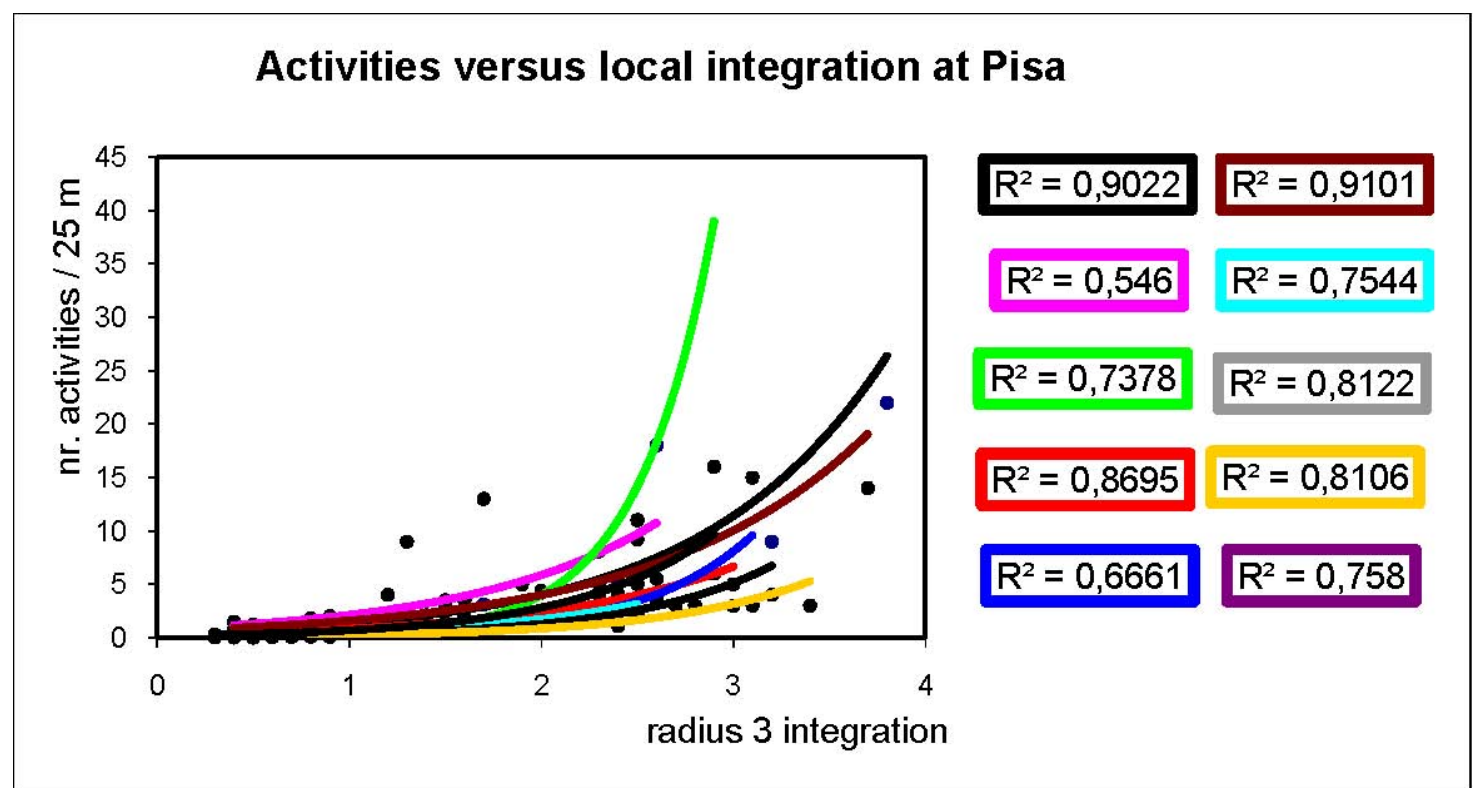

Fig. 3 Correlation of local $(\mathrm{R}=3)$ integration value versus density of activities in the lines of Pisa. Each colour distinguish one of the sub-systems.

tourist attractions). And just schools, universities, hospitals, administrative offices, museums and monuments are precisely what the town of Pisa is mainly made of. As a provement, we may get a look at the correlation integration versus activities mentioned above, and notice a clear lack of correspondence in the subsystem nr. 2 (where $\mathrm{R}^{2}=0.546$ ), due to values of density of activities largely higher than the expectancy resulting on the basis of integration values. As a matter of fact, that area is characterized by the presence of several faculties (Engineering, Chemistry, Pharmacy, Medicine, Foreign Languages, Informatics: on the whole more than 25,000 students) of the University of Pisa, by the presence of an important regional hospital, the Santa Chiara University Hospital, by the presence of relevant military (paratroopers) barracks, and, above all, by the presence of the leaning tower and of the other monuments of the Piazza dei Miracoli (around 2,500,000 visitors in a year). All these activities, undoubtedly monopolistic as lacking in competitors in a wide area around Pisa, of course attract here a large amount of movement and activities (mainly hotels, restaurants, souvenirs shops, bookshops, stationery stores, etc.) although the lines of the area do actually result characterized by poor values of global integration. This obvious evidence do persuade that a PEBA merely oriented onto the configurational pattern of the grid would inevitably disregard the presence of the monopolistic activities, and more in general of the non-configurational ones; what in Pisa should clearly be dramatically wrong and unacceptable, for two main reasons: first, those activities are a large and prominent part of the urban functional structure; moreover the universal accessibility to those activities, mainly to be regarded as public and social services, is strongly requested as a necessary requirement.

It is then clear that the results of the analysis above, worked out by means of configurational techniques, ought to be integrated by a further kind of analysis, based on a functional approach, which is assuming as an input the presence, the size and the specific position of the located activities. More in detail, we decided to integrate the results of configurational analysis, and in particular the distribution of the pure accessibility index, with the outputs of an interactional model, providing the distribution of generalized accessibility; pure accessibility and generalized one, taken together, will then been assumed as a reliable indicator of the crowding of paths and of the consequent necessity of their universal accessibility. On such basis, we have 
assumed as our input the distribution of economic activities in the municipality of Pisa, as in Italy it is provided with a ten-yearly frequency by ISTAT (Istituto Nazionale di Statistica); we have used the data of 2001 (the most recent available data), and the selected values are expressed in the number of occupied employers and spatially referred to the singles census tracts. The whole municipality of Pisa is covered by 918 census tracts, and that partition is sufficiently minute so as to allow a systemic analysis of the settlement by means of a gravitational model. On the basis of the employments data in the census tracts, we have then calculated the distribution of spatial economic potential (the generalized accessibility mentioned above) according to the usual expression

$$
\mathrm{Ea}=\mathrm{K} \Sigma \mathrm{j} \omega \mathrm{j} \mathrm{daj}^{-\gamma}
$$

what obviously implies the processing of a $918 \times 918$ symmetric matrix. We have then organized the results of the processed model by means of a GIS, using as cells the same 918 ISTAT census tracts, thus obtaining and presenting by a chromatic representation the distribution of generalized accessibility in the whole area of the municipality of Pisa, which is here shown in Fig. 4.

The several and strong differences with regard to the distribution of integration (pure accessibility, as we did assert) can be observed by the comparison of Fig. 1 and Fig. 4. The same differences may be easily noticed by the comparison of the spatial economic core with the integration core deducted by the analysis above. In Figs. $5-6$, it's possible to respectively observe the global integration core and the local integration core of Pisa, while in Fig. 7 is planimetrically represented the economic spatial potential of the settlement; in all the mentioned cases, the marked out areas result characterized by values over 90th percentile.

More in detail, we may notice that high values of generalized accessibility (red cells) characterize the areas along the river Arno, as well as the whole north-west area of Pisa, where we find Piazza dei
Miracoli, the Santa Chiara Hospital, the faculties of Engineering, Medicine and Pharmacy; as it can be easily understood, and could be easily predicted, such high values depend on the presence of those prominent activities, and are in contrast with the poor values of integration. On the other hand, all the main global integrators result located within high potential areas, demonstrating that a favourable position on the grid (from a configurational point of view) actually attracts activities and makes the correspondent spatial potential to increase. It's also worth saying that in the processed model we could not take in sufficient account the role of movement attractors of several activities and public services, which result provided with a limited number of employers with respect to their actual importance and attractiveness: here we obviously refer to the monuments of Piazza dei Miracoli, but also to the other churches and monuments of Pisa, as well as to the railway station and to the bus station. Therefore, in all those cases we must consider underestimated the respective spatial potential. At last, also in the interactional approach, as we did above in the configurational method, we have selected and pointed out an accessibility core, which was assumed as characterized by the areas over the 90th percentile of the spatial potential value.

The two different pattern of accessibility can therefore be integrated, so as to point out the urban spaces that, either in a configurational vision or in an interactional approach, result deserving interventions for providing a universal accessibility. The result is a grid of paths, extending from the inner core (where they are, as predictable, far denser) up the edge of the urban settlement, connecting both the configurational sub-centres and the most prominent and attracting located activities.

Obviously, a procedure like that can be regarded as too rough, in that it assumes a strictly binary logic, simply dividing all the urban spaces in two categories and distinguishing them in universally accessible spaces and inaccessible one. A slightly more detailed procedure could instead graduate the several levels of 


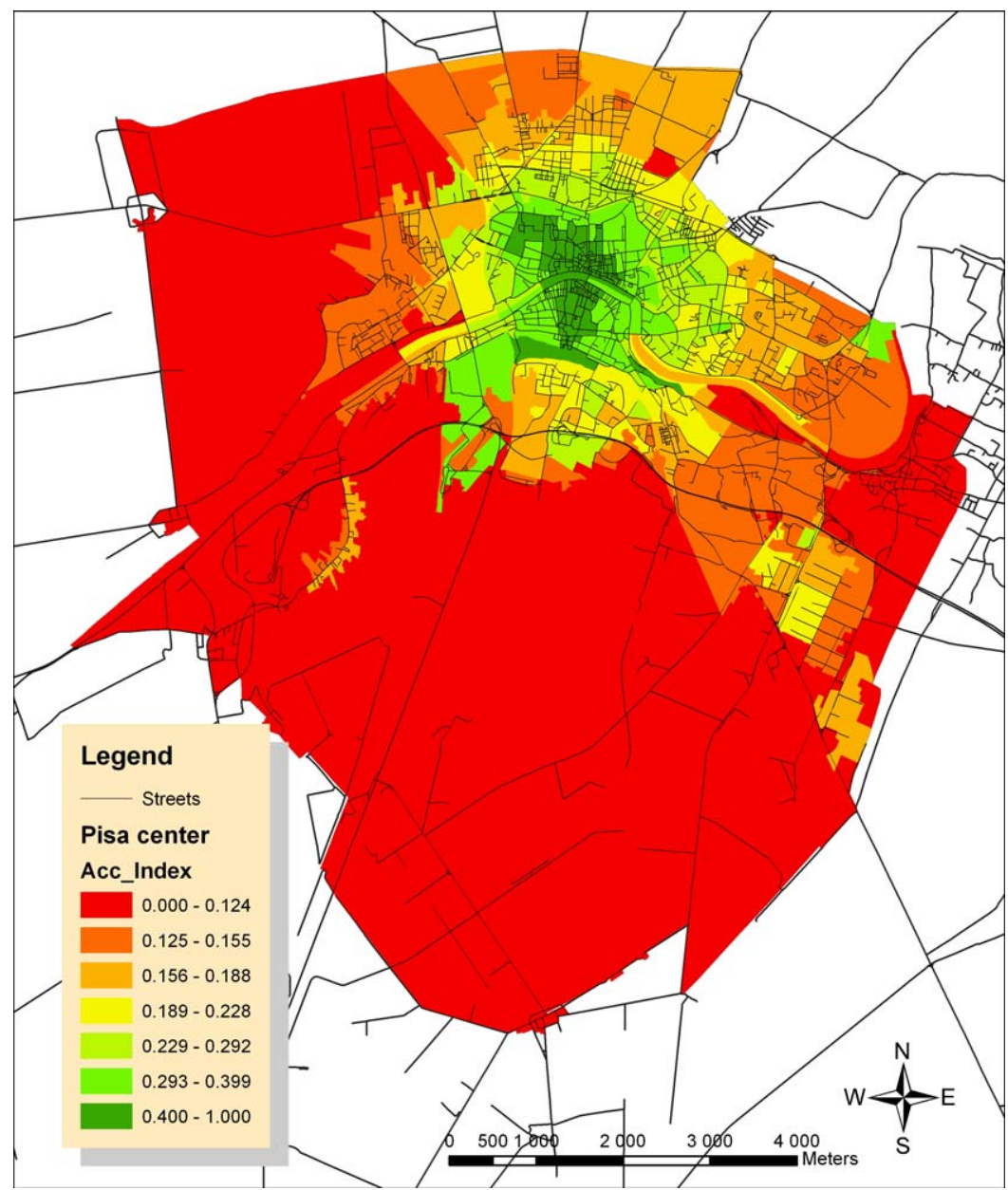

Fig. 4 Chromatic representation of the distribution of economic spatial potential in the whole area of the municipality of Pisa.

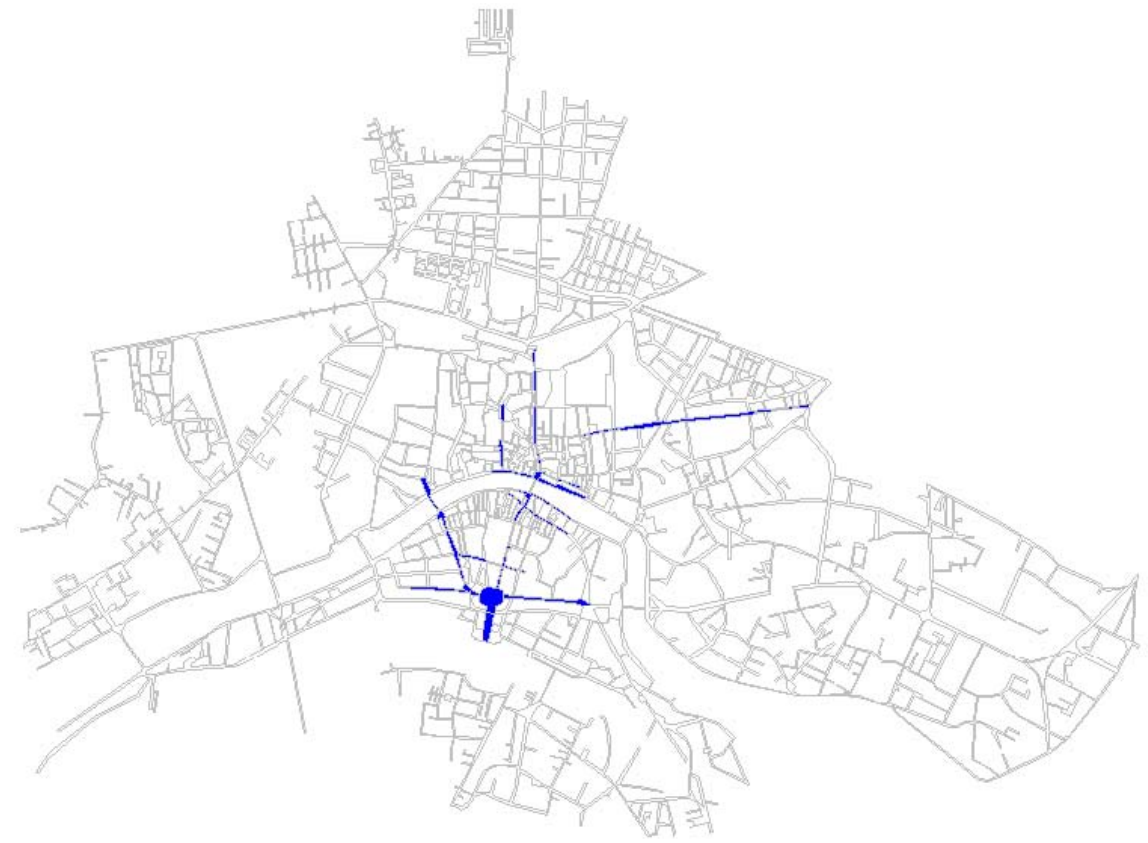

Fig. 5 Global integration core of Pisa. 


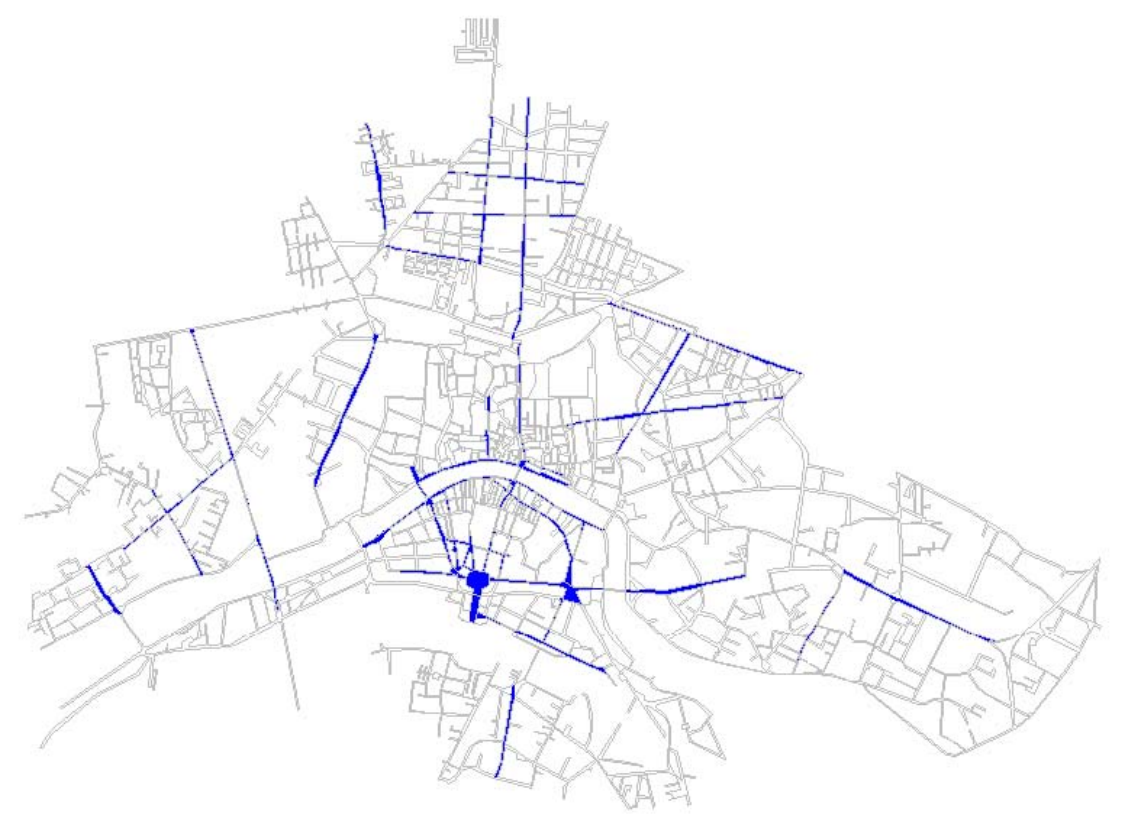

Fig. 6 Local (radius 3) integration core of Pisa.

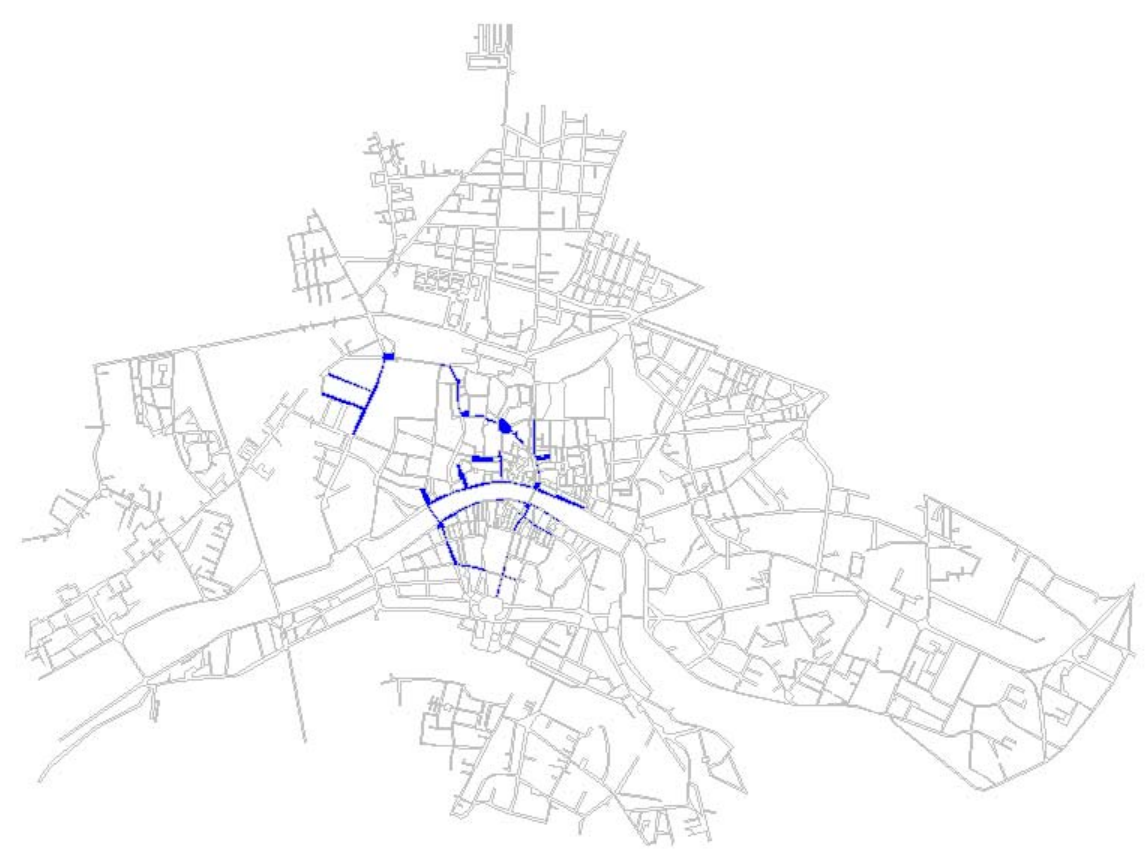

Fig. 7 Economic spatial potential core of Pisa.

material accessibility the urban paths ought to comply with, according to their respective actual configurational/interactional accessibility. As an example, we could define three levels of spatial accessibility requirement, which could respectively be named "universal accessibility requested", "partial accessibility requested" and "accessibility non requested"; here the expression "partial accessibility" could refer to the mere elimination of the architectural obstacles and barriers, so as to just allow disabled people to safety move along the sidewalks (Gallagher, Scott, 1996). In Fig. 8 we can notice the represented result of a subdivision like that in the planimetric layout of Pisa, where the universal accessibility is requested for the paths with an accessibility value over the 90th percentile and the partial accessibility is 


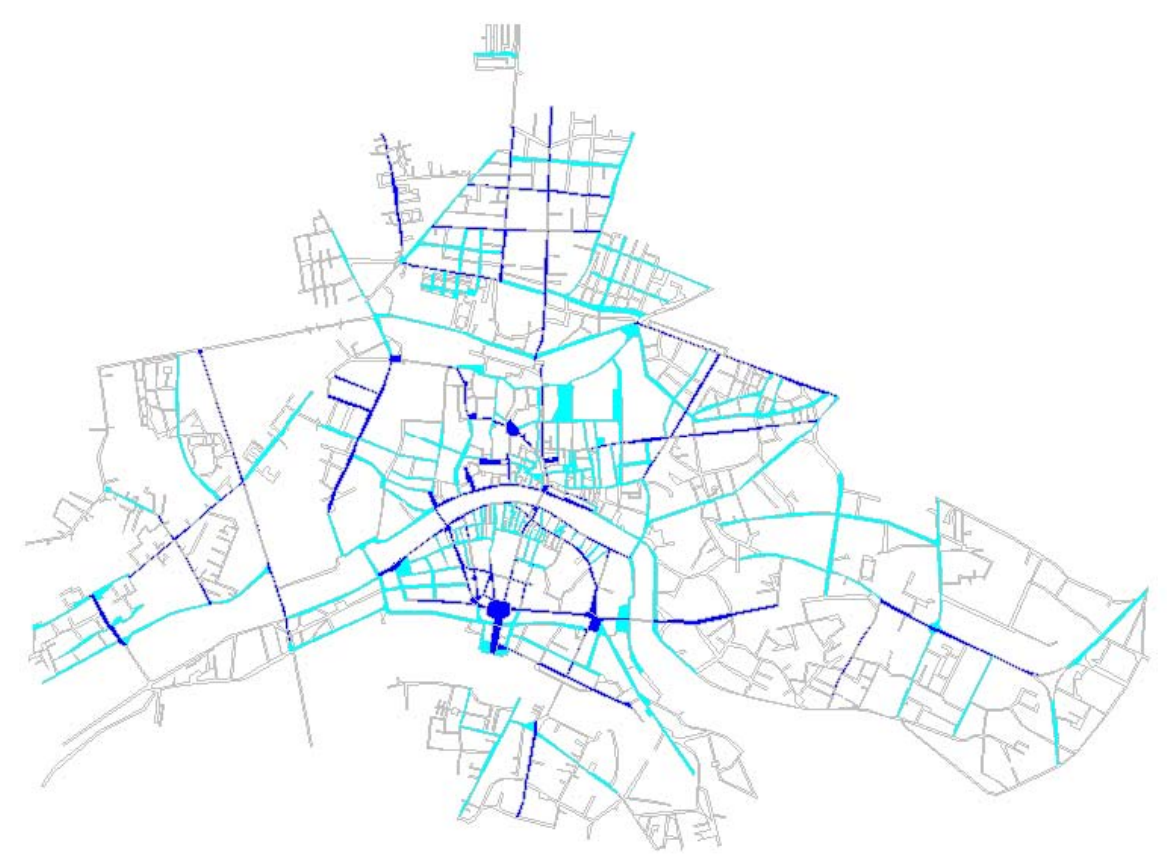

Fig. 8 Proposed classification of the public open spaces of Pisa with reference to the required material accessibility: in blue the universal accessible spaces, in light blue the partial accessible ones.

requested for the lines with an accessibility value from the 50th up to the 90th percentile; in the exposed representation, the universal accessibility is required for the areas which result marked in blue, while the only partial accessibility is required for those marked in light blue. Obviously, the proposed subdivision and the correspondent values are here presented as a mere example, since they can largely vary according to the specific features of the local context (that is the actual presence of obstacles or territorial impediments), to the available funds for the intervention as well as to the political willingness to approach the asymptote of the universal accessibility.

\section{Conclusions}

The configurational approach to the analysis of urban spaces is here applied to support the working out of accessibility plans in order to provide urban planning with an objective decision making support tool as well as to overcome the faults and the limits of the traditional functional vision. Only a configurational approach, in fact, can account for the effects of the grid on the distribution of accessibility, and hence on the distribution of pedestrian movement flows. In such sense, the experimentation of the configurational techniques to the urban case of Pisa, for several reasons here presented as an ideal case study, has provided outstanding results, as it allows to single out a widespread network of paths that, due to their centrality, are likely to get crowded with pedestrian movement and then chiefly needy of intervention for enhancing their material accessibility.

At the same time, those techniques make some physiological limits of the configurational approach to emerge, in that it can't account for the effects on movement of the non-configurational activities, and mainly, among them, of the monopolistic ones, which in Pisa are numerous, conspicuous and strongly attractive; it hence makes clear that the configurational techniques can be suitably integrated by a gravitational model, based on the interaction relations expected to connect all the located activities. The results of the configurational analysis, that is the distribution of pure accessibility (local and global integration values), and the results of the processing of a spatial interaction model, that is the distribution of generalized 
accessibility (economic spatial potential), taken together, indicate the distribution of the actual demand for the material accessibility of the urban spaces. Furthermore, beyond the limits of the matter of the inclusive design of urban spaces, two general results of this research appear worth noticing. First, the concrete application of the configurational techniques as a decision making support tool in the operational urban planning. Second, the integration of the configurational techniques with the traditional interactional analysis, so as to account for the effects of both grid and activities on urban phenomena.

\section{References}

[1] Switzer Jacqueline Vaughn, Disabled Rights: American Disability Policy and the Fight for Equality. Washington, DC: Georgetown University Press, 2003.

[2] Clarkson John, Roger Coleman, Ian Hosking and Sam Walter, Inclusive Design Toolkit, Cambridge: Cambridge Engineering Design Centre, 2004.

[3] Goldsmith Selwyn, Universal Design: A Manual of Practical Guidance for Architects, Oxford: Oxford Architectural Press, 2000.

[4] Preiser Wolfgang and Elaine Ostroff, Universal Design Handbook, New York: McGraw Hill, 2001.

[5] Imrie Rob and Peter Hall, Inclusive Design: Designing and Developing Accessible Environments, London: Spon. 2001.

[6] Clarkson John, Roger Coleman, Simon Keates and Cherie Lebbon, Inclusive Design: Design for the Whole Population, London: Springer, 2003.
[7] Burton Elizabeth and Lynne Mitchell, Inclusive Urban Design: Streets for Life, London: Architectural Press, 2006.

[8] Hillier Bill, Cities as movement economies, Urban Design International 1 (1996) 41-60.

[9] Cutini Valerio, Urban space and pedestrian movement A study on the configurational hypothesis, in: Cybergeo, Revue Européenne de Geographie, $n^{\circ}$ 111, 26.10.1999.

[10] Cutini Valerio, Centrality and Land Use: Three Case Studies on the Configurational Hypothesis, in: Cybergeo, Revue Européenne de Geographie, $n^{\circ}$ 188, 26.03.2001.

[11] Batty Michael, Recent developments in land use modeling: A review of British research, Urban Studies 9 (1972) 151-177.

[12] G. Alan Wilson, Urban and Regional Models in Geography and Planning, London: Wiley, 1974.

[13] G. Alan Wilson, Entropy in Urban and Regional Modelling, London: Pion, 1970.

[14] Alonso William, Location and Land Use, Cambridge: Harvard University Press, 1964.

[15] Bortoli, Michele and Valerio Cutini, Centralità e uso del suolo urbano. L'analisi configurazionale del centro storico di Volterra, Pisa: ETS, 2001.

[16] Cutini Valerio, Configuration and centrality: Some evidence from two Italian case studies, in: Proceedings of the Space Syntax $3^{\text {rd }}$ International Symposium, Atlanta 7-11 May 2001, 32.1-32.11, Alfred Tauban College of Architecture and Urban Planning, University of Michigan. 2001.

[17] Cutini Valerio, Working on the edge of town: The periphery as a spatial pattern in computational science and its applications, ICCSA, Berlin: Springer, 2008, pp. 26-41. 